www.jmscr.igmpublication.org Impact Factor (SJIF): 6.379

Index Copernicus Value: 71.58

ISSN (e)-2347-176x ISSN (p) 2455-0450

crossref DOI: _https://dx.doi.org/10.18535/jmscr/v6i2.19

Journal Of Medical Science And Clinical Research

\title{
Primary Cytoreductive surgery (PS) and Neoadjuvant Chemotherapy (NACT) followed by Interval Debulking Surgery (NACT/IDS) in Advanced Epithelial Ovarian Cancer (EOC): Regional Cancer Centre Experience
} Authors

Dr Shobha Krishnappa ${ }^{1}$, Dr Umadevi. $K^{2}$, Dr Jayashree Natarajan ${ }^{3}$, Dr Rajshekar. S Kunderagi ${ }^{4}$, Dr Pallavi. V. $\mathbf{R}^{5}$, Sri. Vijay C. $\mathbf{R}^{6}$, Dr P. Sridhar ${ }^{7}$, Dr U. D Bafna ${ }^{8}$

1,2,3,4,5,8 Department of Gynaec Oncology, Kidwai Cancer Institute, Bangalore, India

${ }^{6}$ Department of Epidemiology and Biostatistics, Kidwai Cancer Institute, Bangalore, India

${ }^{7}$ Department of Radiation Oncology, Kidwai Cancer Institute, Bangalore, India

Corresponding Author

Dr Rajshekar. S Kunderagi

Department of Gynaec Oncology, Kidwai Cancer Institute, Bangalore, India

\begin{abstract}
Background: The standard treatment of advanced ovarian cancer [FIGO stage 3 and 4] has been complete cytoreductive surgery (CRS) followed by Platinum based chemotherapy. Neoadjuvant chemotherapy (NACT) followed by interval debulking surgery (NACT/IDS) has been considered as an alternative to conventional $P S$ in treating advanced EOC.

Objective: Analysis of survival and prognostic factors in advanced EOC patients who underwent Primary Cytoreductive surgery (PS) with Neoadjuvant Chemotherapy (NACT) followed by Interval Debulking Surgery (NACT/IDS)

Methods: A retrospective analysis of 25 patients who had advanced EOC surgery (stage $3 \& 4$ ) between the years 2005-2009. Clinicopathological, potential prognostic factors and progression-free survival (PFS) were analyzed.

Results: No significant difference was seen in the operative time, blood loss among PS and NACT-IDS group. Need for extensive surgeries which included bowel surgeries, diaphragm stripping/ resection, splenectomy, groin node debulking, pelvic/parietal peritonectomy was higher in PS group (53\%) when compared to NACT-IDS group (35\%).

In optimal cytoreductive group, when patients took complete chemotherapy, the median PFS was 21 months and in patients with incomplete chemotherapy it was 10 months $(P=0.025)$.

In suboptimal cytoreductive group, when complete chemotherapy was taken, the median PFS was 24 months when compared to 6 months in those who took incomplete chemotherapy $(p=0.001)$. There was no difference in overall survival.

Conclusion: Though in many institutes, Primary debulking surgery is still the gold standard treatment. As long as optimal debulking is feasible in EOC, NACT-IDS can be considered for patients where optimal debulking is not possible or surgery is risky due to various patient related/ tumor related factors.

Keywords: Primary Cytoreductive surgery (PS), Neoadjuvant Chemotherapy (NACT), Interval Debulking Surgery (IDS), Epithelial Ovarian Cancer (EOC).
\end{abstract}




\section{Introduction}

According to GLOBOCAN 2012, every year 6.1 per 100,000 new cases of ovarian cancer are seen and 3.7 per 100,000 of these cases succumb to the disease. Ovarian cancer is the leading cause of death among Gynaecologic malignancies. The unfavourable prognosis of this disease is largely due to non specific symptoms and lack of early detection methods. Approximately $70 \%$ to $80 \%$ of the cases are stage III or IV when first diagnosed.

The standard treatment of advanced ovarian cancer [FIGO stage 3 and 4] has been complete cytoreductive surgery (CRS) followed by Platinum based chemotherapy ${ }^{(1)}$. Optimal cytoreductive surgery with residual disease less than 1 or no macroscopic disease is one of the most important prognostic factors to predict survival in patients with advanced epithelial ovarian cancer ${ }^{(2-5)}$.

The definition of optimal debulking has changed since 1980; originally considered to be- no residual disease of greater than $2 \mathrm{~cm}$ in diameter and more recently as residual tumor of $<1 \mathrm{~cm}$. The current aim is to leave, no macroscopic disease. The percentage of advanced EOC patients who are able to undergo optimal cytoreduction varies in the literature from $15-85 \%{ }^{(2-6)}$. Chi et al ${ }^{(3)}$ reported an optimal debulking rate of $76 \%$ with aggressive surgical methods like diaphragmatic stripping/ resection, partial liver resection, cholecystectomy and partial pancreatectomy.

Neoadjuvant chemotherapy (NACT) is a chemotherapy method for primary solid tumors before surgical debulking. Ovarian cancer is a chemo sensitive tumor with a response rate of $70 \%$ to $80 \%^{(7)}$. Thus, NACT can usually result in adequate tumor shrinkage and increase the possibility of optimal tumor debulking. Therefore, NACT followed by interval debulking surgery (NACT/IDS) has been considered as an alternative to conventional PS in treating advanced EOC. This approach is especially useful in cases with poor PS or where optimal debulking is not feasible due to extensive fixed disease.

\section{Aims and Objectives}

1. To compare the clinical characteristics of patients who underwent Primary Cytoreductive surgery (PS) and Neoadjuvant Chemotherapy (NACT) followed by Interval Debulking Surgery (NACT/IDS) in Advanced Epithelial Ovarian Cancer (EOC).

2. To analyse survival in both the arms.

3. To determine prognostic factors based on univariate and multivariate analysis.

\section{Materials and Methods}

\section{Patients and treatment}

This is a retrospective study which included patients who underwent surgery for advanced EOC (stage $3 \& 4$ ) between the years 2005-2009. Totally $276(75.8 \%)$ patients had advanced cancer. Out of them 144 patients (52\%) had primary cytoreductive surgery and 132 patients $(48 \%)$ had NACT and interval debulking surgery. In PS group, out of 144 patients 12 patients were lost for follow up after completion of their treatment protocol and in IDS group 30 patients were lost for follow up. Totally 234 patients were included for analysis (132 in PS and 102 in NACT-IDS group).

Neo-adjuvant chemotherapy was administered after cytological or histological confirmation of malignancy. NACT was administered in stage 4 disease (27 patients), poor performance status (ECOG >2, 20 patients), gross ascites with hypo albuminemia, extensive upper abdominal deposits (37 patients) and fixed pelvic mass (18 patients). 2 to 3 cycles of Platinum with or without Paclitaxel based NACT was given. 70 patients received single platinum agent and 32 patients received combined taxane and platinum agent.

Response to NACT was assessed by WHO criteria 2 weeks after last CT and was followed by IDS. 55 patients $(54 \%)$ had complete response, 38 (37\%) had partial response and 9 patients $(9 \%)$ had stable/progressive disease after NACT.

Three patients were declared inoperable in PS group due to extensive peritoneal carcinomatosis 
and 1 patient in IDS group due to dense adhesions in abdomen and pelvis.

Standard debulking surgeries were performed in both groups which included hysterectomy, bilateral salpingo-oophorectomy, omentectomy, pelvic/para aortic node debulking, debulking of metastatic deposits. Optimal cytoreduction was considered when the residual disease was $<1 \mathrm{~cm}$.

After primary surgery, patients received 6 cycles of either single agent platinum or combined with taxol adjuvant chemotherapy. After interval debulking surgery, patients received 3-4 cycles of adjuvant chemotherapy. Six cycles was considered as complete chemotherapy.

All patients were followed up every 3 months by clinical examination, ultrasonography and CA-125 levels for 2 years and subsequently once in 6 months. The interval between complete treatment and occurrence of disease (image, cytological and histological evidence) were taken as progression free survival.

\section{Statistical analysis}

Progression free survival (PFS) was analyzed using the Kaplan-Meier method. Multivariate forward stepwise cox regression by including age, tumor histology, tumor grade, residual disease, adjuvant chemotherapy, complete therapy was used to analyze the survival.

\section{Results}

Patient characteristics are shown in Table-1. Operative details: No significant difference was seen in the operative time, blood loss among PS and NACT-IDS group. Need for extensive surgeries which included bowel surgeries, diaphragm stripping/ resection, splenectomy, groin node debulking, pelvic/parietal peritonectomy was higher in PS group (53\%) when compared to NACT-IDS group (35\%). Complications from these extensive surgeries are also more in PS group. Details of these variables can be seen in Table $2 \& 3$.

\section{Survival analysis in PS group}

Median progression free survival (PFS) was 17 months and median overall survival 41 months. Log rank test showed no difference in the PFS between the serous and non serous tumors. Grade 2 tumors had a PFS of 24 months when compared to grade 3 tumors that had 15 months. Though there was an increase in PFS, it was statistically insignificant $(p=0.08)$. The median PFS for patients who had optimal cytoreduction was 19 months and those with suboptimal surgery was 15 months $(\mathrm{P}=0.25)$. The median PFS for patients who took complete adjuvant chemotherapy was 21 months compared to those who took incomplete chemotherapy was 9 months, the result is statistically significant at $5 \%$ level of significance $(\mathrm{P}=0.001)$.

In optimal cytoreductive group, when patients took complete chemotherapy, the median PFS was 21 months and in patients with incomplete chemotherapy it was 10 months $(\mathrm{P}=0.025)$. In suboptimal cytoreductive group, when complete chemotherapy was taken, the median PFS was 24 months when compared to 6 months in those who took incomplete chemotherapy $(p=0.001)$. There was no difference in OS.

\section{Survival analysis in NACT-IDS group}

The mean follow up in this group was 40 months with median PFS and OS of 16 and 45 months. There was no significant difference in PFS with respect to tumor grade. 55 (54\%) patients had complete response, $38(37 \%)$ patients had partial response and 9 (9\%) patients had stable/ progressive response to NACT. In patients who had complete response, the median PFS was 28 months when compared to patients who had partial response whose median PFS was 10 months $(\mathrm{P}=0.001)$. In patients who had stable/progressive disease, median PFS was 6 months $(\mathrm{P}=0.001)$. Median PFS in patients who took single agent $\mathrm{CT}$ was 14 months and for those with combination $\mathrm{CT}$ was 17 months $(\mathrm{P}=0.347)$. The median PFS for patients with optimal cytoreduction was 23 months and for patients with 
suboptimal cytoreduction was 10 months $(\mathrm{P}=<0.001)$. Median PFS for patients who took complete chemotherapy was 17 months and for the patients who took incomplete chemotherapy it was 12 months $(\mathrm{P}=0.08)$.

In the optimal cytoreduced group, for patients who took complete CT, the median PFS was 30 months and 28 months for the patients who took incomplete CT. In the suboptimal cytoreduced group, the median PFS in patients who took complete CT was 18 months and 15 months in patients who took incomplete $\mathrm{CT} .(\mathrm{P}=\mathrm{ns})$.
Multivariate forward stepwise cox regression by including age, tumor histology, tumor grade, residual disease, adjuvant chemotherapy and complete chemotherapy has resulted in prediction of PFS by complete chemotherapy only in PS group $(\mathrm{P}=0.001)$. In NACT-IDS group multivariate analysis has shown prediction for PFS by NACT response $(0.001)$, residual disease $(\mathrm{P}=0.001)$ and by complete chemotherapy $(\mathrm{P}=0.03)$. There was no impact on OS.

Table 1: Variables of cases in both the groups

\begin{tabular}{|l|c|c|c|}
\hline SI.No & VARIABLES & Primary Surgery & NACT-IDS \\
& & n=132 (56\%) & n=102 (44\%) \\
\hline $\mathbf{1}$ & Age & 48.39 years & 51.52 years \\
\hline $\mathbf{2}$ & FIGO stage & & \\
& Stage 3 & 127 & 75 \\
& Stage 4 & 05 & 27 \\
\hline $\mathbf{3}$ & Histology & & $80 \%$ \\
& Serous & $77.3 \%$ & $20 \%$ \\
\hline $\mathbf{4}$ & Non serous & $22.7 \%$ & $1(1 \%)$ \\
& Grade & $10(8 \%)$ & $22(22 \%)$ \\
& 1 & $36(27 \%)$ & $79(77 \%)$ \\
\hline
\end{tabular}


Table 2: Need for various surgical procedures in both groups

\begin{tabular}{|c|c|c|c|c|}
\hline Sl. No & Surgical Procedures & $\begin{array}{r}\text { PS } \\
\mathrm{n}=132(56 \%)\end{array}$ & $\begin{array}{r}\text { NACT-IDS } \\
\mathrm{n}=102(44 \%)\end{array}$ & P Value \\
\hline 1 & Bowel surgeries & $29(22 \%)$ & $10(10 \%)$ & 0.049 \\
\hline 2 & Diaphragm stripping & $17(13 \%)$ & $02(2 \%)$ & 0.0024 \\
\hline 3 & Splenectomy & $04(3 \%)$ & $00(0 \%)$ & 0.000 \\
\hline 4 & Pelvic peritonectomy & $20(15 \%)$ & $12(12 \%)$ & 0.45 \\
\hline \multirow[t]{2}{*}{5} & Groin node debulking & 00 & $01(1 \%)$ & \\
\hline & Total & 70 & 25 & \\
\hline
\end{tabular}

Table 3: Variables \& complications in both groups

\begin{tabular}{|c|c|c|c|}
\hline Sl. No & Surgical Procedures & $\begin{array}{c}\text { PS } \\
\mathrm{n}=132(56 \%)\end{array}$ & $\begin{array}{l}\text { NACT-IDS } \\
n=102(44 \%)\end{array}$ \\
\hline 1 & Mean operative time (range) & 3.2 hours ( $2-5$ hours) & 2.43 hours (1.5-4.5 hours) \\
\hline 2 & Mean blood loss & $400 \mathrm{ml}$ & $300 \mathrm{ml}$ \\
\hline 3 & Mean blood transfusion required & $250 \mathrm{ml}$ & $200 \mathrm{ml}$ \\
\hline 4 & $\begin{array}{c}\text { Residual disease } \\
<1 \mathrm{~cm} \\
>1 \mathrm{~cm}\end{array}$ & $\begin{array}{c}107(81 \%) \\
25(19 \%)\end{array}$ & $\begin{array}{l}89(87 \%) \\
13(13 \%)\end{array}$ \\
\hline 5 & $\begin{array}{c}\text { Post op complications } \\
\text { Bowel injury } \\
\text { Bladder injury } \\
\text { Major vessel injury } \\
\text { Hypotensive shock } \\
\text { DIC } \\
\text { Post op Sepsis } \\
\text { Wound infection/ dehiscence } \\
\text { Burst abdomen } \\
\text { Mortality* }\end{array}$ & $\begin{array}{l}03 \\
02 \\
00 \\
03 \\
00 \\
01 \\
05 \\
02 \\
03\end{array}$ & $\begin{array}{l}00 \\
00 \\
01 \\
03 \\
01 \\
00 \\
03 \\
00 \\
01\end{array}$ \\
\hline
\end{tabular}




\section{Discussion}

In majority of the institutes, PDS followed by adjuvant chemotherapy is still the standard of care for advanced stage EOC patients. The amount of residual tumor after PDS is an important prognostic factor for survival in several retrospective and prospective studies ${ }^{(2,8)}$. Thus, great efforts including radical surgical procedures have been made to achieve optimal cytoreduction. But in the present study, out of 132 patients, $81 \%$ optimal cytoreduction has been achieved in PS group.

Primary cytoreduction has been performed in 52\% of advanced ovarian cancer in our institute. In a selected subgroup of patients $48 \%$ who were medically unfit for PS or where optimal cytoreduction would not be able to achieve, they received NACT (2-3 cycles) followed by interval debulking surgery.

It was demonstrated in many studies that NACTIDS effectively increases the feasibility of optimal cytoreductive surgery in advanced $\mathrm{EOC}^{(9-12)}$. In our study also, we were able to achieve optimality similar to primary group though the performance status was poor and disease was extensive in patients who received NACT group compared to the primary surgery group. The median PFS is 17 and 16 months in PS and NACT-IDS group respectively which was statistically insignificant. However, the most important question on whether NACT-DS is superior to PDS in survival is still under investigation.

The majority of the studies showed that NACTIDS had no impact on OS compared with PDS, including a meta-analysis of 21 studies and a recently published randomized trial $(9,10,13,14)$. NACT-IDS was found to have similar survival $(11,12,15)$. The present study being retrospective study, there has been heterogeneity in two groups; we are able to achieve similar results in the selected subgroup of patients where optimal resection would not have been feasible with upfront surgery.

Although no survival advantage is offered by NACT-IDS, this treatment method provides favourable perioperative results. In our study, the patients treated with NACT-IDS had lesser perioperative complications and lesser need for more radical surgeries. These results are consistent with previous studies ${ }^{(15)}$.

Though the NACT-IDS group included higher staged tumors and large volume tumor which in itself is a negative prognostic factor, the PFS among both the groups is comparative. Response to NACT, complete debulking is a significant factor in determining PFS in patients of NACT group. The completion of $\mathrm{CT}$ is a significant factor in determining the PFS in patients of PS group.

\section{Conclusion}

Though in many Institutes, primary debulking surgery is still the gold standard treatment as long as optimal debulking is feasible in EOC. In our institute, NACT is an option considered for patients where optimal debulking would not have been possible or surgery is risky due to various patient related/ tumor related factors. After NACT we were able to achieve similar optimal cytoreduction as in primary surgery. The PFS is similar in both the groups. Optimal debulking and completing the regimen of $\mathrm{CT}$ are important factors in determining the PFS in NACT and PS groups respectively. The guidelines for selection of cases for NACT and time of intervention need to be standardized in larger randomized studies to determine overall survival.

\section{References}

1. Griffiths CT, Fuller AF. Intensive surgical and chemotherapeutic management of advanced ovarian cancer. SurgClin North Am. 1978 Feb;58(1):131-42.

2. Bristow RE, Tomacruz RS, Armstrong DK, Trimble EL, Montz FJ. Survival effect of maximal cytoreductive surgery for advanced ovarian carcinoma during the platinum era: a meta-analysis. $\mathrm{J}$ ClinOncol Off $\mathrm{J}$ Am SocClinOncol. 2002 Mar 1;20(5):1248-59. 
3. Chi DS, Eisenhauer EL, Zivanovic O, Sonoda Y, Abu-Rustum NR, Levine DA, et al. Improved progression-free and overall survival in advanced ovarian cancer as a result of a change in surgical paradigm. GynecolOncol. 2009 Jul;114(1):26-31.

4. Aletti GD, Dowdy SC, Gostout BS, Jones MB, Stanhope CR, Wilson TO, et al. Aggressive surgical effort and improved survival in advanced-stage ovarian cancer. Obstet Gynecol. 2006 Jan;107(1):77-85.

5. Lim MC, Kang S, Song YJ, Park SH, Park SY. Feasibility and Safety of Extensive Upper Abdominal Surgery in Elderly Patients with Advanced Epithelial Ovarian Cancer. J Korean Med Sci. 2010 Jul;25(7):1034-40.

6. Du Bois A, Reuss A, Pujade-Lauraine E, Harter P, Ray-Coquard I, Pfisterer J. Role of surgical outcome as prognostic factor in advanced epithelial ovarian cancer: a combined exploratory analysis of 3 prospectively randomized phase 3 multicenter trials: by the Arbeitsgemeinschaft Gynaekologische Onkologie Studiengruppe Ovarialkarzinom (AGOOVAR) and the Groupe d'Investigateurs Nationaux Pour les Etudes des Cancers de l'Ovaire (GINECO). Cancer. 2009 Mar 15;115(6):1234-44.

7. McGuire WP, Hoskins WJ, Brady MF, Kucera PR, Partridge EE, Look KY, et al. Cyclophosphamide and cisplatin compared with paclitaxel and cisplatin in patients with stage III and stage IV ovarian cancer. N Engl J Med. 1996 Jan 4;334(1):1-6.

8. Elattar A, Bryant A, Winter-Roach BA, Hatem M, Naik R. Optimal primary surgical treatment for advanced epithelial ovarian cancer. Cochrane Database Syst Rev. 2011;(8):CD007565.

9. Rauh-Hain JA, Rodriguez N, Growdon WB, Goodman AK, Boruta DM 2nd, Horowitz NS, et al. Primary debulking surgery versus neoadjuvant chemotherapy in stage IV ovarian cancer. Ann SurgOncol. 2012 Mar;19(3):959-65.

10. Morice P, Dubernard G, Rey A, Atallah D, Pautier P, Pomel C, et al. Results of interval debulking surgery compared with primary debulking surgery in advanced stage ovarian cancer. J Am Coll Surg. 2003 Dec;197(6):955-63.

11. Kuhn W, Rutke S, Späthe K, Schmalfeldt B, Florack G, von Hundelshausen B, et al. Neoadjuvant chemotherapy followed by tumor debulking prolongs survival for patients with poor prognosis inInternational Federation of Gynecology and Obstetrics

12. Hou JY, Kelly MG, Yu H, McAlpine JN, Azodi M, Rutherford TJ, et al. Neoadjuvant chemotherapy lessens surgical morbidity in advanced ovarian cancer and leads to improved survival in stage IV disease. Gynecol Oncol. 2007 Apr;105(1):211-7.

13. Everett EN, French AE, Stone RL, Pastore LM, Jazaeri AA, Andersen WA, et al. Initial chemotherapy followed by surgical cytoreduction for the treatment of stage III/IV epithelial ovarian cancer. Am J Obstet Gynecol. 2006 Aug;195(2):568-574; discussion 574-576.

14. Lee S-J, Kim B-G, Lee J-W, Park C-S, Lee $\mathrm{J}-\mathrm{H}$, Bae D-S. Preliminary results of neoadjuvant chemotherapy with paclitaxel and cisplatin in patients with advanced epithelial ovarian cancer who are inadequate for optimum primary surgery. J Obstet Gynaecol Res. 2006 Feb;32(1):99-106.

15. Rafii A, Deval B, Geay J-F, Chopin N, Paoletti X, Paraiso D, et al. Treatment of FIGO stage IV ovarian carcinoma: results of primary surgery or interval surgery after neoadjuvant chemotherapy: a retrospective study. Int J Gynecol Cancer Off J IntGynecol Cancer Soc. 2007 Aug;17(4):777-83. 